\title{
Progress toward the clinical application of patient-specific pluripotent stem cells
}

\author{
Evangelos Kiskinis and Kevin Eggan
}

The Stowers Medical Institute, Harvard Stem Cell Institute, Department of Stem Cell and Regenerative Biology, Harvard University, Cambridge, Massachusetts, USA.

\begin{abstract}
Induced pluripotent stem (iPS) cells are generated by epigenetic reprogramming of somatic cells through the exogenous expression of transcription factors. These cells, just like embryonic stem cells, are likely to have a major impact on regenerative medicine, because they self-renew and retain the potential to be differentiated into all cell types of the human body. In this Review, we describe the current state of iPS cell technology, including approaches by which they are generated and what is known about their biology, and discuss the potential applications of these cells for disease modeling, drug discovery, and, eventually, cell replacement therapy.
\end{abstract}

\section{Introduction}

Human ES cells, which are derived from the inner cell mass of blastocyst stage embryos, have the unique ability to self-renew indefinitely while maintaining the potential to give rise to all cell types in the human body (1). Induced pluripotent stem (iPS) cells share these salient characteristics of ES cells but are instead generated via reprogramming of somatic cells through the forced expression of key transcription factors (2). The seminal achievement of induced pluripotency holds great promise for regenerative medicine. Patient-specific iPS cells could provide useful platforms for drug discovery and offer unprecedented insights into disease mechanisms and, in the long term, may be used for cell and tissue replacement therapies.

The successful cloning of animals such as Dolly the sheep in $1997(3,4)$ and the subsequent derivation of human ES cells in 1998 (1) brought forward the concept of therapeutic cloning, in which pluripotent ES cell lines tailored to the genetic makeup of specific individuals might provide a plentiful source of therapeutic cells (5). Although significant advancements toward this goal have been made $(6,7)$, successful somatic cell nuclear transfer (SCNT) (a technique whereby the DNA of an unfertilized egg is replaced by the DNA of a somatic cell) with human cells remains elusive and is fraught with social and logistical concerns. Alternative methods for deriving pluripotent cells, such as cell fusion (8) and culture-induced reprogramming (9), have been developed, but these approaches still suffer from severe practical and technical limitations. In contrast, the generation of pluripotent cells by exogenous expression of transcription factors circumvents many previous limitations, as this approach is not technically demanding and does not require embryonic material or oocytes. We therefore believe that iPS cell technology will have a significant impact on regenerative medicine, and in this article we review current methodologies used for generating iPS cells and then discuss their potential clinical applications.

\section{iPS cells: state of the art}

The arrival of iPS cells. In the first report of defined factor reprogramming (10), Kazutoshi Takahashi and Shinya Yamanaka reprogrammed mouse fibroblasts through retroviral transduction

Conflict of interest: The authors have declared that no conflict of interest exists. Citation for this article: J. Clin. Invest. 120:51-59 (2010). doi:10.1172/JCI40553. with 24 transcription factors highly expressed in ES cells. This cadre of genes was gradually reduced to four that encode the transcription factors octamer 3/4 (Oct4), SRY box-containing gene 2 (Sox2), Kruppel-like factor 4 (Klf4), and c-Myc (10). The resulting iPS cells were selected based on their ability to express the gene F-box protein 15 (Fbx15), which is specifically expressed in mouse ES cells and early embryos. Although the selected cells were similar to ES cells in morphology, growth properties, and ability to form teratomas (neoplasmic tumors characterized by the presence of cells corresponding to all three embryonic germ layers) in immunodeficient mice, they differed in terms of global gene expression profiles and certain DNA methylation patterns. Perhaps the most important difference was that these cells failed to produce adult chimeric mice. This might be because although Fbx15 is specifically expressed in mouse ES cells and embryos, it is dispensable for maintaining pluripotency and mouse development (11). In subsequent studies (12-15), when improved end points for the reprogramming process were selected, such as the expression of Nanog and Oct4, the resulting iPS cells were even more similar to ES cells, could contribute to adult chimeras, and were transmitted through the germ line.

Shortly after these reprogramming successes in the mouse, Yamanaka used the human orthologs of the four transcription factor-encoding genes to generate iPS cells from human fibroblasts (2). Concurrently, two other groups achieved similar reprogramming of human somatic cells using slightly different combinations of genes that also included OCT4 and SOX2 (see Table 1 for details) $(16,17)$. Within months, it had been proven that it was possible to derive iPS cells from patients suffering from the neurodegenerative disease amyotrophic lateral sclerosis (ALS) (18) as well as patients with other diseases, including juvenileonset type 1 diabetes mellitus, Parkinson disease (PD) (19), and spinal muscular atrophy (SMA) (20).

Mechanism of reprogramming. Given that all cells within an organism have the same genome, the functional characteristics of different cell types are defined by specific patterns of gene expression. Epigenetic molecular mechanisms control gene transcription by inducing stable changes in gene expression. These changes favor the formation of either an accessible or inaccessible chromatin state without directly affecting the DNA sequence (21).

Developmental programming establishes gene expression patterns that are set and maintained via histone modifications and 
Table 1

Mouse and human iPS cells have been generated in a variety of ways

\begin{tabular}{|c|c|c|c|c|c|c|c|}
\hline $\begin{array}{l}\text { Cell } \\
\text { type }\end{array}$ & $\begin{array}{l}\text { Reprogramming } \\
\text { factors }\end{array}$ & $\begin{array}{l}\text { Method of } \\
\text { delivery }\end{array}$ & $\begin{array}{l}\text { Timeline } \\
\text { (wk) }\end{array}$ & Efficiency & $\begin{array}{l}\text { Genomic } \\
\text { integration }\end{array}$ & $\begin{array}{l}\text { Genomic } \\
\text { integration } \\
\text { removed }\end{array}$ & Refs. \\
\hline \multicolumn{8}{|l|}{ Mouse } \\
\hline MEFs & $0, \mathrm{~S}, \mathrm{~K}, \mathrm{M}$ & Retroviral vectors & $2-3$ & $0.01 \%-0.050 \%$ & Yes & No & $10,12-15$ \\
\hline MEFs & $0, S, K$ & Retroviral vectors & $3-4$ & $0.001 \%-0.01 \%$ & Yes & No & $56,65,98$ \\
\hline $\begin{array}{l}\text { Hepatocytes and } \\
\text { stomach cells }\end{array}$ & $0, \mathrm{~S}, \mathrm{~K}, \mathrm{M}$ or $\mathrm{O}, \mathrm{S}, \mathrm{K}$ & Retroviral vectors & $2-3$ & $0.5 \%-3 \%$ & Yes & No & 46 \\
\hline Neural stem cells & $\begin{array}{c}0, \mathrm{~S}, \mathrm{~K}, \mathrm{M} \text { or } \mathrm{O}, \mathrm{S}, \mathrm{K} \\
\text { or } \mathrm{O}, \mathrm{K}, \mathrm{M} \text { or } \mathrm{O}, \mathrm{S}, \mathrm{M}\end{array}$ & Retroviral vectors & $1-4$ & $0.1 \%-5 \%$ & Yes & No & 48,99 \\
\hline Neural stem cells & $0, \mathrm{~K}$ or $0, \mathrm{M}$ or $\mathrm{O}$ & Retroviral vectors & $2-4$ & $0.1 \%-0.2 \%$ & Yes & No & 32,100 \\
\hline B lymphocytes & $0, S, K, M$ & Lentiviral vectors ${ }^{A}$ & $2-3$ & $0.01 \%-0.1 \%$ & Yes & No & 34 \\
\hline Hepatocytes & O,S,K,M & Adenoviral vectors & $4-5$ & $0.0001 \%-0.001 \%$ & No & - & 29 \\
\hline MEFs & $0, S, K, M$ & Plasmid transient transfection & $3-4$ & $0.0001 \%-0.001 \%$ & No & - & 30 \\
\hline MEFs & O,S,K,M & piggyBac transposon ${ }^{A}$ & 2 & NR & Yes & Yes & 67 \\
\hline MEFs & O,S,K,M & Nonviral plasmid transfection & 2 & $2.5 \%$ & Yes & Yes & 66 \\
\hline MEFs & 0,S,K,M or $0, S, K+V P A$ & Recombinant proteins & $4-5$ & $0.002 \%-0.008 \%$ & No & - & 31 \\
\hline MEFs & 0,K + Bix and BayK & Retroviral vectors & $2-3$ & $0.007 \%-0.02 \%$ & Yes & No & 60 \\
\hline MEFs & O,S,K,M & Plasmid nucleofection & $2-3$ & NR & No & - & 68 \\
\hline MEFs, TTFs & $0, \mathrm{~S}, \mathrm{~K}, \mathrm{M}$ & Single lentiviral vector ${ }^{A}$ & $2-3$ & $0.5 \%$ & Yes & No & 63 \\
\hline MEFs, TTFs & $\begin{array}{c}0, \mathrm{~K}, \mathrm{M}+\text { RepSox or } \\
\text { O,K + RepSox }\end{array}$ & Retroviral vectors & 2 & $\sim 1 \%$ & Yes & No & 61 \\
\hline \multicolumn{8}{|l|}{ Human } \\
\hline $\begin{array}{l}\text { Skin fibroblasts } \\
\text { and bone marrow } \\
\text { mesenchymal cells }\end{array}$ & $\begin{array}{c}0, \mathrm{~S}, \mathrm{~K}, \mathrm{M} \text { or } \mathrm{O}, \mathrm{S}, \mathrm{K}, \mathrm{M}, \mathrm{T}, \mathrm{SV} \\
\text { or } \mathrm{O}, \mathrm{S}, \mathrm{K}, \mathrm{M}, \mathrm{N} \text { or } \mathrm{O}, \mathrm{S}, \mathrm{K}\end{array}$ & Retroviral vectors & $2-5$ & $0.001 \%-1 \%$ & Yes & No & $2,16,18,19$ \\
\hline Skin fibroblasts & $0, S, K$ or $0, S+V P A$ & Retroviral vectors & 4 & $0.001 \%-0.01 \%$ & Yes & No & 57 \\
\hline Keratinocytes & $0, S, K, M$ or $0, S, K$ & Retroviral vectors & $1-2$ & $1 \%$ & Yes & No & 49 \\
\hline Peripheral blood cells & $0, S, K, M$ & Retroviral vectors & 2 & $0.01 \%-0.02 \%$ & Yes & No & 50 \\
\hline $\begin{array}{l}\text { Skin fibroblasts } \\
\text { and keratinocytes }\end{array}$ & $0, \mathrm{~S}, \mathrm{~K}, \mathrm{M}$ or $\mathrm{O}, \mathrm{S}, \mathrm{K}, \mathrm{M}, \mathrm{N}$ & Lentiviral vectors ${ }^{A}$ & $3-4$ & $0.002 \%$ & Yes & No & 101 \\
\hline Skin fibroblasts & O,S,N,L or O,S,N,L,M,K & Lentiviral vectors & $2-3$ & $0.01 \%-1 \%$ & Yes & No & $17,20,33$ \\
\hline Skin fibroblasts & $\mathrm{O}, \mathrm{S}, \mathrm{K}, \mathrm{M}$ or $\mathrm{O}, \mathrm{S}, \mathrm{K}$ & Lentiviral vectors ${ }^{A}$ & $3-5$ & $N R$ & Yes & Partial ${ }^{B}$ & 36 \\
\hline Embryonic fibroblasts & $0, S, K, M$ & piggyBac transposon ${ }^{A}$ & $2-4$ & NR & Yes & No & 67 \\
\hline Embryonic fibroblasts & $0, \mathrm{~S}, \mathrm{~K}, \mathrm{M}$ & piggyBac transposon ${ }^{A}$ & $2-4$ & $0.005 \%-0.01 \%$ & Yes & No & 66 \\
\hline Skin fibroblasts & 0,S,N,L,M,K,SV & Episomal vectors & $N R$ & $0.003 \%-0.006 \%$ & No & - & 33 \\
\hline Adipose stem cells & O,S,K,M & Lentiviral vectors & $2-3$ & $0.2 \%$ & Yes & No & 51 \\
\hline Skin fibroblasts & $0, \mathrm{~S}, \mathrm{~K}, \mathrm{M}$ & Recombinant proteins ${ }^{C}$ & 8 & $0.001 \%$ & No & - & 32 \\
\hline
\end{tabular}

ADox-inducible systems. BViral LTR is not excised. CProteins were delivered in the form of whole cell extracts. O, Oct4; S, Sox2; K, Klf4; M, c-Myc; N, Nanog; L, Lin28; SV, SV4OLT; T, hTert; MEF, mouse embryonic fibroblast; TTF, tail tip fibroblast; NR, not reported; -, not relevant.

DNA methylation (22). This is a one-way process (reversed only in germ cells) that gradually leads to somatic cell types with reduced pluripotency (for more detailed reviews, see refs. 22, 37) (Figure 1). Transcription factors are the key regulators of this process (23), and there can be no better evidence for their imperial role in determining the functional characteristics of a cell than their ability to reprogram differentiated adult cells to a pluripotent state $(2,10)$. Different combinations of just six transcription factor-encoding genes, Oct4, Sox2, Klf4, c-Myc, Nanog, and Lin28, have been used to achieve reprogramming in mouse and human cells $(2,10,17)$. Of these, Oct4 (24), Sox2 (25), and $\operatorname{Nanog}(26,27)$ were known to encode proteins functionally important in maintaining pluripotency in embryos and ES cells; the rest encode proteins with functions in a wider variety of cell types. Determining the exact functional role of each of the proteins encoded by the six genes in the reprogramming process is an active area of investigation, although a recent genome-wide analysis of promoter occupancy at various stages of iPS cell generation has shown that they co-occupy regulatory regions of a number of genes associated with pluripotency (28).

Initial protocols for making iPS cells utilized viral vectors to exogenously express the four transgenes, which integrated in the genome of somatic cells. However, it is now possible to induce iPS cells with non-integrating methods in mouse (29-31) and human cells $(32,33)$, and although these methods are not yet efficient, they have clearly demonstrated that random insertional mutagenesis is not an essential mechanistic requirement for reprogramming. Furthermore, successful reprogramming of terminally differentiated postmitotic cells such as B lymphocytes (34) has definitively shown that reprogramming does not require multipotent target cells that may exist within somatic cell populations.

ES versus $i P S$ cells. A number of studies have clearly demonstrated that iPS cells are highly similar to ES cells $(12-15,28,35-37)$. Most of the iPS cell lines that have been generated have a normal karyotype and possess telomeres with characteristics of those in 


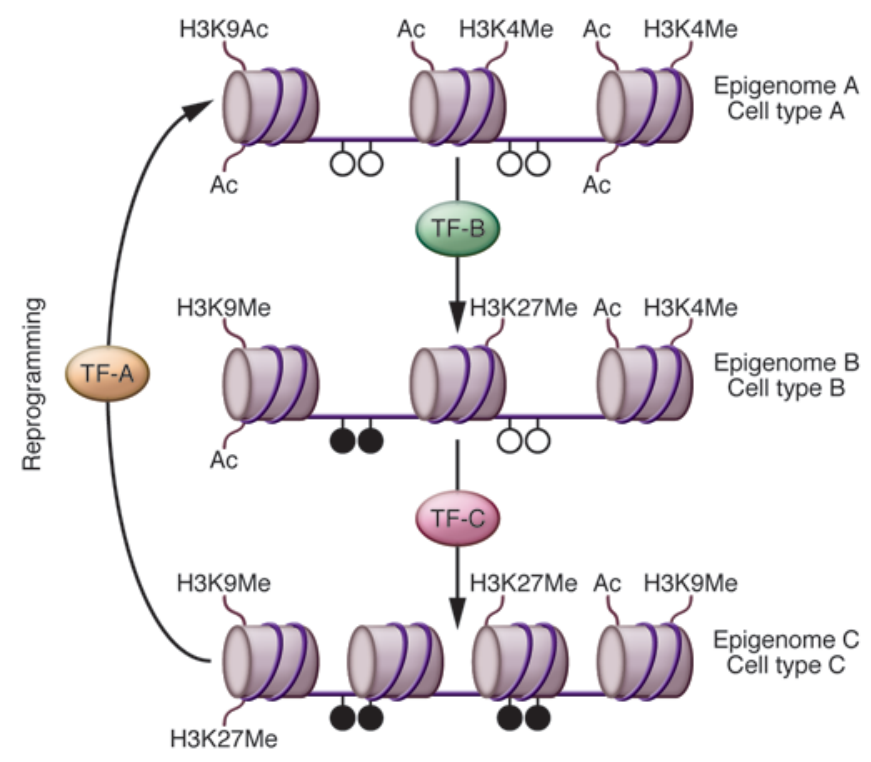

ES cells (38). In terms of the epigenetic state of iPS cell lines, the promoters of pluripotency genes have no DNA methylation, while female mouse iPS cells reactivate the inactive $\mathrm{X}$ chromosome and exhibit random inactivation upon differentiation (12). Furthermore, the promoters occupied by the reprogramming proteins in iPS cells are highly congruous to those occupied by the endogenous proteins in ES cells, and this correlates with closely related global gene expression profiles (28).

However, small differences in gene expression patterns between iPS and ES cells exist (39). The nature of these differences is not yet well understood, although genomic integration of transgenes has been shown to be a contributing factor. Soldner et al. recently partially addressed the issue of integration by demonstrating that upon excision of the reprogramming transgenes (but not the viral long-terminal repeats [LTRs]), the genome-wide expression profile of iPS cells generated from human fibroblasts correlated more closely with that of ES cells than it did prior to excision: only 48 genes remained substantially different in their expression in contrast to 271 before excision (36). Similarly, when Yu et al. generated human iPS cells without any detectable genomic integration, only a small set of genes remained differentially expressed when compared with human ES cells (33).

The reason for the remaining differences in the global transcriptome between ES and iPS cells remains unclear, although the unique genetic makeup of each line may contribute to a certain degree, as has been shown for human lines (40) and mouse ES cell lines (41). It is noteworthy that a recent report indicates that iPS cells (generated by retroviral genomic integration) exhibit a unique gene and miRNA expression profile that gradually becomes more similar to that of ES cells with extended in vitro culturing (39). Moreover, this iPS cell-specific expression signature is at least partially conserved among iPS cells generated in independent reprogramming experiments and even in integration-free iPS cell lines (39). These issues can be best resolved by comparing the expression profiles of an ES line and an iPS cell line of the same genetic background.

While the value of comparing iPS and ES cell lines is unquestionable, the functional ramifications of small differences between iPS

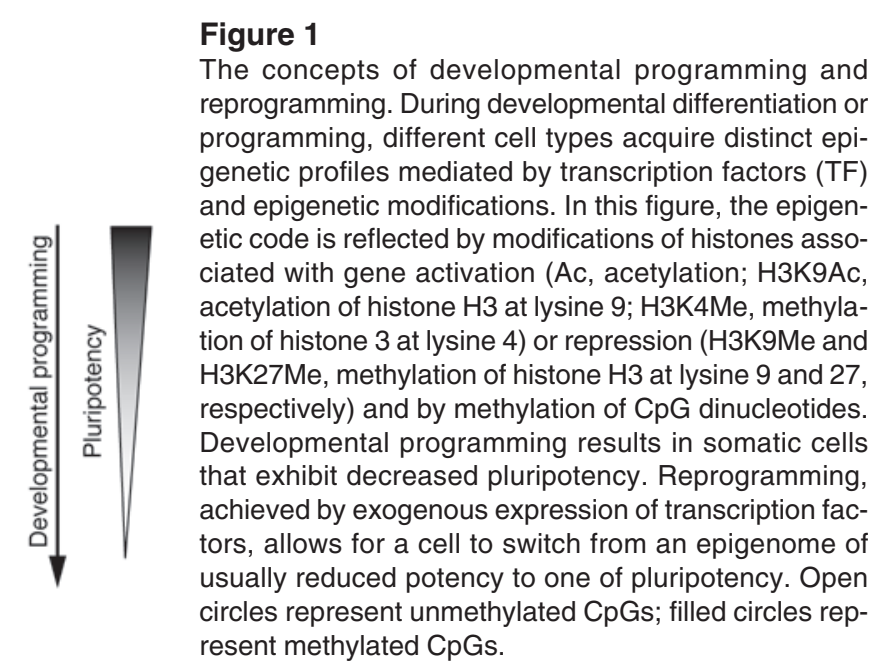

and ES cells in terms of global gene expression levels and epigenetic state remain unclear. Moreover, it remains unknown whether the observed differences will result in functional variation that interferes with the utility of certain iPS cell lines.

The ability of mouse iPS cells to generate an entire mouse, as was recently shown via tetraploid complementation assays (a technique in which iPS cells are injected into tetraploid blastocysts) (42-44), and of human iPS cells to form teratomas in vivo (19) indicates in the most stringent tests that they are pluripotent cells and suggests that the defined factor reprogramming approach produces cells with a developmental potential similar to that of ES cells. To our knowledge, no conspicuous differences in the efficiency of differentiation between iPS and ES cells toward specific lineages have been reported. Although these experiments are difficult to perform due to the heterogeneity of the resulting cell populations, they will be a key indicator of whether iPS cells can be readily used for a variety of practical applications, including disease modeling.

What is the best way of making iPS cells? First-generation iPS cells were generated by retroviral transduction $(2,10)$. Since then, the technique has been optimized and reproduced in a number of different ways (Table 1 and references therein). The most important variables include choice of cell type to reprogram, choice of the cocktail of reprogramming genes, and method for gene transfer (Figure 2). Nimet Maherali and Konrad Hochedlinger recently wrote an excellent review of protocols, highlighting the details of different methodologies to make iPS cells, and we therefore cover this area only briefly (45).

Embryonic fibroblasts (MEFs) and tail-tip fibroblasts (TTFs) in the mouse and dermal fibroblasts in the human have been the most widely used cell types to reprogram, largely due to their availability and ease of accessibility. In addition, various other cell types have also been reprogrammed, including hepatocytes (29), stomach cells (46), B lymphocytes (34), pancreatic $\beta$ cells (47), and neural stem cells (48) in the mouse; keratinocytes (49), mesenchymal cells (19), peripheral blood cells (50), and adipose stem cells (51) in the human; and melanocytes in both species (52). Variable efficiencies and kinetics of the process have been described, while the in vitro age of the cell type (passage number) correlates inversely with 
Reprogramming factors/delivery method Oct4, Sox2, Klf4, c-Myc, Nanog, Lin28

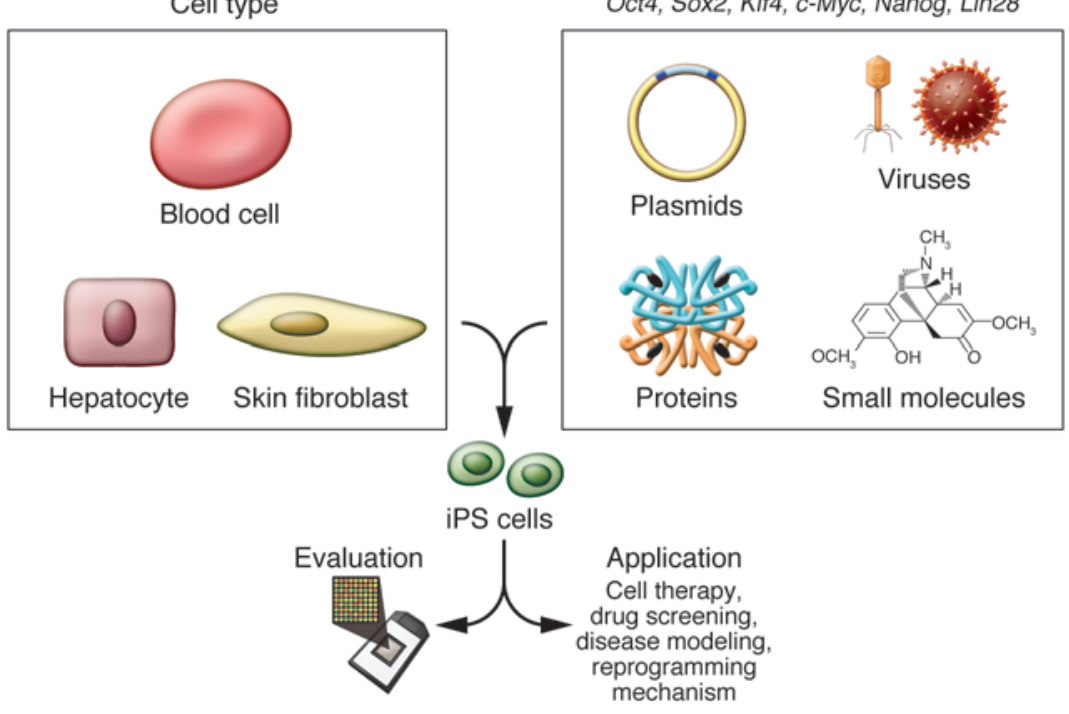

\section{Figure 2}

Generation of iPS cells. The choice of the cell type from which to derive iPS cells, the choice of reprogramming factors and methods of delivery, as well as evaluation of iPS cell progeny, will depend on the potential application of the resulting cell types. the efficiency of reprogramming (53). The same inverse correlation has been effectively demonstrated for the differentiation stage of target cells, with mouse hematopoietic stem and progenitor cells being more efficiently reprogrammed than terminally differentiated $\mathrm{B}$ and $\mathrm{T}$ lymphocytes (54).

A recent study addressed the variability in the teratoma-forming propensity of cells differentiated from iPS cell lines generated from a number of mouse tissues (55). Miura et al. found that neurospheres (nonadherent spherical clusters of neural stem and progenitor cells) derived from iPS cells generated from MEFs formed teratomas with efficiency similar to that of neurospheres derived from ES cells, while neurospheres derived from iPS cells generated from either TTFs or hepatocytes formed teratomas more readily. These results were associated with the presence of greater numbers of residual undifferentiated iPS cells in neurospheres derived from iPS cells generated from either TTFs or hepatocytes, and they can be used to indirectly assess the variation in differentiation efficiency that might arise as a result of the target cell that is reprogrammed.

Of the original four transcription factor-encoding genes, $c-M y c$ has been shown to be dispensable for reprogramming in the mouse and human, while Klf4 and Sox2 have been shown to be dispensable in reprogramming strategies utilizing cell types that endogenously express them (see Table 1). Oct4 is the only factor that cannot be replaced by other family members and the only one that has been required in every reprogramming strategy in either mouse or human cells.

Importantly, small molecules, including DNA methyltransferase inhibitors $(35,56)$, the histone deacetylase inhibitor valproic acid (VPA) $(56,57)$, and the histone methyltransferase inhibitor BIX-01294 (58), have been shown to substantially improve the efficiency of reprogramming, even in cases without inclusion of exogenous Klf4 and c-Myc (for VPA) or exogenous Sox2 and c-Myc (for BIX-01294). In addition, the compound kenpaullone can compensate for the reprogramming factor Klf4 (59), while a combination of BIX-02194 and BayK8644 can compensate for Sox2 (60), even in cells that do not endogenously express it, although the mechanism(s) by which these small molecules function remain unclear. We have also recently identified a small molecule inhibitor of TGF- $\beta$ signaling that replaces Sox 2 by inducing Nanog expression (61). Of note, it remains to be determined whether full reprogramming through the exclusive use of chemicals is possible.

iPS cells have been generated using a number of different gene transfer methods, including retro-, lenti-, and adenoviral vectors and nonviral plasmids, and recently by direct recombinant protein delivery. The use of genome-integrating viral vectors such as retroviruses and lentiviruses results in iPS cells that would be inappropriate for therapeutic use, as even a single insertional mutation $(62,63)$ and the potential for reactivation of viral transgenes substantially increase the risk that transplanted cells would become transformed $(14,64,65)$. The major advantage of retroviral vectors is that they are known to undergo progressive silencing in ES cells, while lentiviral systems remain active. However, in certain cases, probably due to the site of genomic integration, retroviral vector expression is maintained $(18,19)$. Recent studies have reported on the generation of iPS cells using genomic integration systems such as lentiviruses (36), plasmids (66), and transposons (67), all of which allow for subsequent transgene removal through the Crelox system or transposases.

More importantly, iPS cell generation has now been achieved without genomic integration. This has been done using adenoviruses (29), repeated plasmid transfection $(30,68)$, and recombinant proteins (31) in the mouse and via the use of episomal vectors (33) and recombinant proteins (32) in the human. If the ultimate use of the iPS cells requires cells free of genomic integrations, then these methods are likely to be preferred over the classic retroviral and lentiviral transduction systems.

Much of the focus of recent research has understandably been on the generation of clinically applicable iPS cells free of viruses and transgenic integrations. We believe that it is now critically important that iPS cells generated by distinct methods are carefully assessed for their variability, stability, and differentiation potential as well as the quality and long-term survival of differentiated cells derived from them. Ultimately, iPS cells generated by each method will need to be examined in detail at the genomic, epigenomic, and functional level in order to determine which reprogramming methods are safe for clinical cell therapy. 


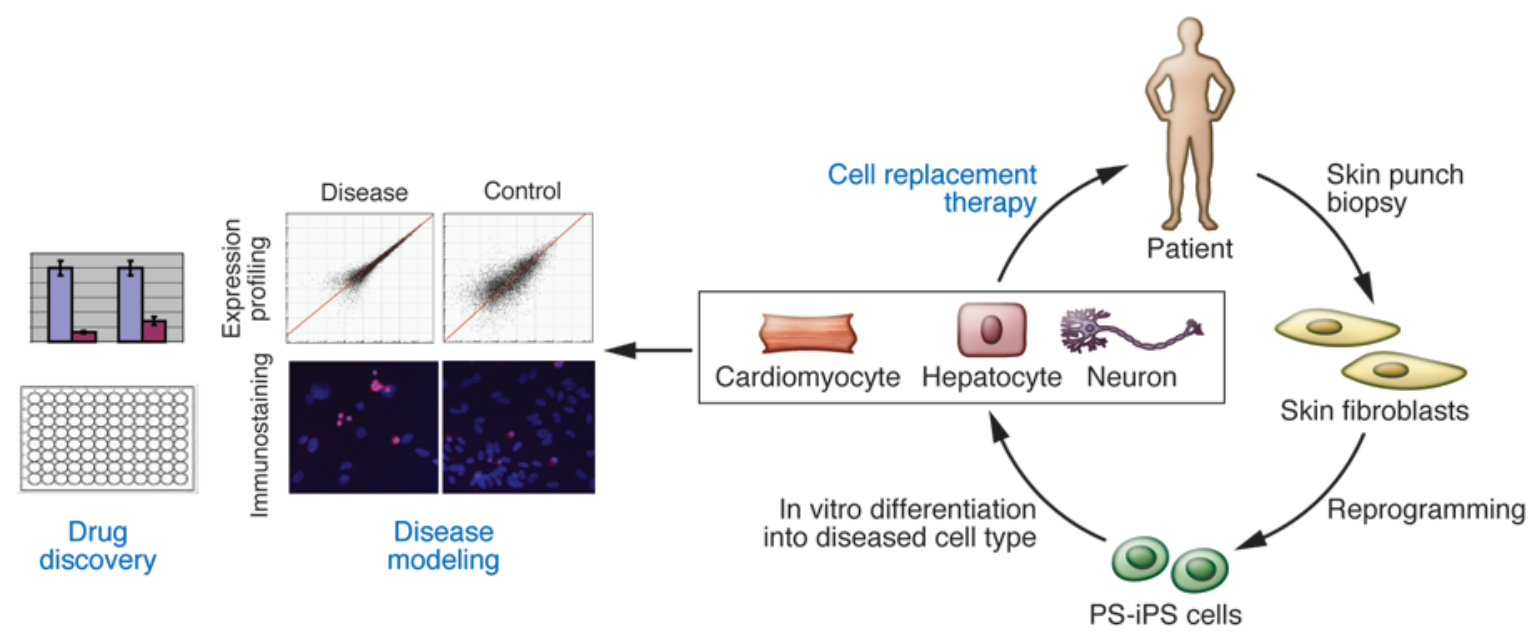

Figure 3

Clinical relevance of iPS cells. iPS cell technology has exciting potential applications in disease modeling and drug discovery. Cell replacement therapy with healthy iPS cell-derived cells is also a possible future development. Genetic mutations can be targeted by gene therapy approaches before or after reprogramming.

Although iPS cells generated using one or two factors rather than four or using recombinant proteins rather than viral expression systems may be clinically safer, it has yet to be demonstrated that this is the case. In addition, the logistical, financial, and practical aspects of each technique will need to be taken into account. It is likely that how one reprograms cells will be determined by the intended usage of the reprogrammed cells. As things currently stand, for example, the fastest and most efficient method to create human iPS cell lines is through lenti- and retroviral transduction. If it is shown that transgene integration sites do not substantially affect the differentiation potential and status of cells types relevant for disease, then these methods might be the preferred approaches for generating iPS cells for use in large-scale drug-screening programs and disease modeling.

An important and controversial issue regarding iPS cell derivation is the standard by which their pluripotent potential is evaluated $(45,69,70)$. We believe that the intended application of the iPS cells should determine the evaluation method. It may be that a neurobiologist who wants to generate motor neurons from patient-specific iPS (PS-iPS) cells should be concerned more with determining the ability of the iPS cells to generate cells that are functionally equivalent to motor neurons found in vivo than whether they are truly pluripotent. The issue of pluripotency becomes more important for studies of the functional mechanism of reprogramming. However, a reproducible, inexpensive, and rapid method to determine the quality of newly established iPS cell lines is urgently required. If it cannot be found, the cost of fully characterizing every new cell line may substantially slow progress. Perhaps an epigenetic and gene expression signature that selectively defines fully reprogrammed iPS and ES cell lines can be identified. This then might enable high-throughput screening of newly derived cell lines.

\section{Clinical relevance}

Cell replacement therapy. The convergence of stem cell research with medical application has long been a source of excitement for the scientific community and the general public alike. Plu- ripotent stem cells offer the hope for treatment of individuals suffering from cellular degeneration caused by either disease or injury. ES cells have already been successfully differentiated in vitro into various therapeutically relevant cell types, including motor and dopaminergic neurons, oligodendrocytes, cardiomyocytes, and hematopoietic precursor cells (71). More importantly, the therapeutic potential of these ES cell-derived somatic cells has been effectively demonstrated in animal models. For example, ES cell-derived hematopoietic precursors have been used to treat immunodeficient mice (7); human ES cell-derived retinal photoreceptors have been used to improve visual functions in blind mice (72); and human ES cell-derived dopaminergic neurons have been shown to reverse some functional deficits in a rat model of PD $(73,74)$. Translation of such therapeutic approaches to human patients has, however, been relatively slow. Although the first clinical trial using ES cell-derived cells, specifically ES cell-derived oligodendrocytes for the treatment of acute spinal cord injury, was approved by the US FDA in January 2009 (75, 76); the trial was put on hold in April 2009 and at the time of writing (November 2009) remained on hold.

The development of cell replacement therapies using ES celldifferentiated cells is, however, burdened with social and religious concerns regarding the use of human embryos, as well as issues involving immune rejection of the transplanted cells. The ability to generate PS-iPS cells by direct reprogramming of human fibroblasts overcomes these barriers and has brought the realization of personalized regenerative medicines closer (Figure 3). PS-iPS cells tailored to specific individuals should provide the opportunity for cell replacement therapy without the need for immunosuppressants, as autologous transplantation of genetically identical cells, and potentially tissues and organs, overcomes the issue of immune rejection. Importantly, in cases of a known genetic defect, utilizing gene therapy approaches could, in principle, restore cellular function.

Shortly after the first report describing iPS cell methodology, a seminal proof-of-principle study demonstrated the potential of iPS cell-based cell replacement therapy in a humanized mouse model 
of sickle cell anemia (77). Hanna et al. reprogrammed fibroblasts from an anemic mouse, corrected the mutant gene by homologous recombination, successfully differentiated the resulting iPS cells into hematopoietic progenitors, and subsequently transplanted these back into the mouse. As a result of the treatment, pathologic features of the disease were substantially ameliorated.

More recently, a similar approach was taken with human individuals with Fanconi anemia, a disease characterized by severe genetic instability (53). In this case, the mutant gene was replaced using lentiviral vectors prior to epigenetic reprogramming of the patient fibroblasts and keratinocytes, as the genetic instability of the mutant fibroblasts made them nonpermissive for iPS cell generation. Importantly, these iPS cells could be differentiated into hematopoietic progenitors as efficiently as ES and wild-type iPS cells, stably maintaining the disease-free phenotype in vitro.

Although these two studies demonstrate the potential of iPS cell technology for treating genetic diseases, they also highlight the urgency for developing better methods of gene therapy, as genetic integration after lentiviral delivery of therapeutic transgenes may be oncogenic (64), and the efficiency of homologous recombination in ES and iPS cells remains extremely low (78). Toward this goal, recent advancements with zinc finger nucleases are rather promising (79).

Studies have also illustrated therapeutic transplantation applications for healthy iPS cell-derived somatic cells in mouse models of disease. In particular, iPS cell-derived dopamine neurons functionally integrated into adult brain in a rat model of PD, leading to an improvement of the phenotype (80), while hemophilia A mice injected with iPS cell-derived endothelial cells into their livers were protected in a death-inducing bleeding assay (81).

iPS cell-based therapies may be particularly important for sporadic forms of diseases such as ALS or PD. The etiology of such forms of these diseases is unclear, but it is thought that they arise from complex interactions between genetic and environmental factors (82). Environmental factors, including toxic pesticides and metals, general lifestyle, and dietary habits have been associated with increased risk of disease $(83,84)$ and are likely to be mirrored in epigenetic alterations. During the reprogramming process, the epigenetic landscape of cells is redrawn, as a terminally differentiated cell is transformed into a pluripotent one. The extent to which the epigenetic profile is altered during reprogramming and the stability of epigenetic imprints during in vitro cell culture remain to be determined.

In cases where a sporadic form of a disease is solely due to epigenetic alterations, iPS cell-derived somatic cells could be therapeutic, as the reprogramming process should reverse the diseasecausing epigenetic modifications. In contrast, if specific genotypes are the root cause of a disease, reprogramming will not alleviate the problem, and the resulting iPS cell-derived somatic cells may succumb to the same degenerative processes that occurred in the patient. A way to address whether epigenetic causes of disease can be corrected in iPS cell-derived cells would be to generate iPS cells from an individual affected by a disease known to be caused by specific epigenetic defects such as myelodysplastic syndrome (85), although it may be that the results will vary according to diseasespecific epigenetic alterations.

iPS cell technology offers the unique opportunity to assess the quality of disease-relevant cell types by directly comparing cells derived in vitro with their genetically identical in vivo counterparts. For instance, if the aim is to transplant iPS cell-derived blood cells back into an individual, there will be an ability to determine how similar the cells derived in vitro are to blood cells isolated from that same individual.

The rapid advancements in the field of iPS cell production during the last three years have led to the generation of clinically relevant cell lines free of genomic integration and oncogenes. Challenges that remain for clinical reprogramming are now more limited to technical issues, such as increasing the efficiencies of iPS cell generation using non-integrating methods, as well as creating them under good manufacturing practice (GMP) conditions.

However, there remain substantial challenges for clinical implementation of the actual therapeutic preparations of cells made from ES and iPS cells. First, it is critical that any ES or iPS cell-derived cell preparation destined for in vivo transplantation be free of undifferentiated cells, as these may form tumors. Second, challenges also lie in efficiently differentiating and purifying populations of disease-relevant cell types. These are in addition to the development of methods for correct delivery of cells into patients and achieving sufficient functional engraftment into the corresponding tissue.

In many instances, such as ALS, where motor neuron degeneration takes place, cell therapy may prove to be extremely challenging, as even if long-term engraftment of ES or iPS cell-derived motor neurons can be achieved, correct projections of neuronal axons to the periphery are unlikely to occur. In these cases, the reprogrammed cells may be best deployed to model the condition in question and eventually identify a small molecule therapeutic that can slow or halt degeneration $(20,86)$.

Disease modeling and drug discovery. Although further work needs to be done toward generating and extensively characterizing "clinical grade" iPS cells before human cell replacement therapies can be attempted, disease modeling and drug screening are two immediate applications for reprogramming technology (Figure 3). While animal models have been crucial in the investigation of disease mechanisms, fundamental developmental, biochemical, and physiological differences exist between mice and humans. The importance of utilizing human cells for these purposes is evidenced by the large numbers of failed clinical trials, which are at least partly attributed to these species differences (87).

The concept of utilizing ES and now iPS cells to model a disease in a culture dish is based on the unique capacity of these cells to continuously self-renew and their potential to give rise to all cell types in the human body $(71,88)$. Thus, pluripotent cells could provide a limitless reservoir of cell types that in many cases were not previously possible to obtain, for example, the motor and dopaminergic neurons affected in ALS and PD. In the past few years, there have been studies reporting successful in vitro modeling of diseases through either the overexpression of known disease-causing genes, such as superoxide dismutase (SOD1) in the case of ALS $(89,90)$ and nuclear receptor-related 1 (NURR1) in the case of PD (91), or the derivation of ES cell lines from preimplantation embryos genetically diagnosed as harboring mutations causing diseases such as fragile X syndrome (92).

While these methods remain attractive, they are limited in their scope because they can only be employed in cases where the genetic defect of a disease is documented. The overwhelming advantage of iPS cell technology is that it allows for the generation of pluripotent cells from any individual in the context of his or her own particular genetic identity, including individuals with sporadic forms of disease and those affected by complex multifactorial diseases of unknown genetic identity, such as autism spectrum disorders (93) and type 1 diabetes (94). 
In the past two years, a number of studies have reported the successful generation of PS-iPS cell lines from individuals with any one of a number of diseases; however, effective disease modeling has only been demonstrated on two occasions so far. In the first study, Ebert et al. reported the differentiation of iPS cell-derived motor neurons from a patient diagnosed with a genetic form of SMA, a neurodegenerative disease that leads to loss of lower motor neurons (20). Importantly, this study was the first to demonstrate a disease-related in vitro phenotype in iPS cell-derived cells. Although motor neurons derived from the PS-iPS cells were initially similar in morphology and number to those derived from wild-type iPS cells, their numbers and size selectively declined after eight weeks in culture. Furthermore, these cells exhibited a deficiency in survival of motor neuron (SMN) protein aggregates, which is a characteristic phenotype associated with SMA. Importantly, this deficiency in SMN levels could be reversed with drug treatment, providing a basis for future drug screens.

In the second study, Lee et al. effectively demonstrated the potential of iPS cell technology to model disease pathogenesis and treatment by creating iPS cell lines from patients with familial dysautonomia (FD), a neuropathy caused by a point mutation in the IкB kinase complex-associated protein (IKBKAP) gene (86). This mutation leads to a tissue-specific splicing defect that was recapitulated in iPS cell-derived tissues. The authors went on to show diseasespecific defects in neurogenesis and migration of neural crest precursors, tissues that were previously unobtainable. These diseasespecific phenotypic changes were then assayed after treatment with candidate drugs, one of which had a beneficial effect.

Although these data are encouraging, both SMA and FD are early-onset genetic disorders, and it may be more challenging to recapitulate other diseases in vitro, such as late-onset sporadic forms of ALS and PD. The average age of onset for these forms of ALS and PD (which account for more than $90 \%$ of all cases) is approximately greater than 50 years, and therefore in vitro cell culture periods of weeks or months may be too short for a pathologic phenotype to arise. Indeed, Soldner et al. differentiated dopaminergic neurons from iPS cells generated from individuals diagnosed with sporadic PD equally as efficiently as from those generated from healthy individuals and did not report any phenotypic differences (36). Importantly, however, although it takes many years for the pathological features of these diseases to become evident, the disease process might be initiated much earlier, and it is possible that analysis of iPS cell-derived motor and dopaminergic neurons might identify more subtle early phenotypic changes in ALS and $\mathrm{PD}$, respectively. Alternatively, certain cell-stressing signals such as hypoxia or neurotoxins may be utilized to facilitate disease pathogenesis in vitro and reveal a potential increased sensitivity in patient-derived cells.

Another challenge to in vitro disease modeling might arise when non-cell-autonomous effects are implicated in disease manifestation. In such cases, production of more than one disease-relevant cell type may be required and coculture assays devised.

We believe that a critical issue regarding iPS cell-based disease modeling assays and therapeutic screens that remains to be resolved is the quantity of appropriate control iPS cell lines that should be included. In order to address this issue, the variability among wildtype iPS cells derived from different individuals and among multiple iPS cell lines generated from the same genetic source needs to be assessed. For example, factors such as the age and sex of the individuals from which iPS cell lines are generated might affect how closely the iPS cell-derived cells model the disease; and the location of the transgene integration sites and transgene reactivation might also affect the efficiency with which PS-iPS cells differentiate into disease-relevant cell types or affect the disease phenotype. Until these questions are properly assessed, it will remain important that multiple iPS cell lines are used as controls.

The arrival of iPS cell-derivation methods has opened exciting avenues for disease modeling and drug discovery. However, we believe that current tools used for these applications, such as animal models and transgenic ES cell lines overexpressing disease-associated genes, remain essential. The application of these tools for effective disease modeling has been proven $(89-91,95,96)$ and should be used in tandem with PS-iPS cells to confirm findings. In this sense, reprogramming technologies are another arrow in the scientist's quiver that can be used to devise and test novel therapies.

Predictive toxicology and pharmacology. The unique properties of ES and iPS cells also provide for practical approaches in pharmaceutical toxicology and pharmacogenomics. In particular, hepatotoxicity and cardiotoxicity are two principal causes of drug failure during preclinical testing, while the variability in individual responses to potential therapeutic agents is also a major problem in effective drug development $(87,97)$. The advantage of iPS cell technology is that it allows for the first time the generation of a library of cell lines that may to a substantial extent represent the genetic and potentially epigenetic variation of a broad spectrum of the population. The use of this tool in high-throughput screening assays could allow better prediction of the toxicology caused by and therapeutic responses induced by newly developed dugs and offer insight into the underlying mechanisms. The net result of this approach would substantially decrease the risk and cost associated with early-stage clinical trials and could lead toward a more personalized approach in drug administration.

\section{Concluding remarks}

Since the first description of iPS cell generation three years ago, there has been remarkable progress toward clinical implementation of reprogramming technologies. The recent successes in iPS cell derivation without viral vectors and genomic integration from human cells has brought the realization of the therapeutic potential of iPS cell technology closer than ever. Importantly, however, the suitability of individual iPS cell derivation methods for generating cell populations for cell replacement therapy, disease modeling, and drug discovery remains to be widely demonstrated, and studies assessing the equivalence of different types of iPS cells are eagerly anticipated. Moreover, extensive characterization of the functionality of iPS cell-derived somatic cells and their functional equivalence with in vivo counterparts needs to be widely demonstrated. The application of the benefits that iPS cells offer is also limited by the ability to derive disease-relevant somatic cells, and major challenges remain in defining pathways that efficiently lead to pure and functional populations of many disease-relevant cells. Given the scientific effort and significant achievements of the past few years, we are hopeful that iPS cell technology will have a positive impact on therapeutic interventions.

\section{Acknowledgments}

We are grateful to Justin Ichida for critical reading of this manuscript, Gabriella Boulting and Alexander Meissner for helpful discussions, and all members of the Eggan Lab for continued support. We also thank Kit Rodolfa and Esther Son for help in cre- 
ating figures. E. Kiskinis is supported by the European Molecular Biology Organization (EMBO). K. Eggan is a fellow of the John D. and Catherine T. MacArthur Foundation and is supported by the Stowers Medical Institute, the Howard Hughes Medical Institute, Project ALS, the New York Stem Cell Foundation, and the Harvard Stem Cell Institute.
Address correspondence to: Kevin Eggan, Harvard University, 7 Divinity Avenue, Cambridge, Massachusetts 02138, USA. Phone: (617) 496-5611; Fax: (617) 384-8234; E-mail: keggan@scrb. harvard.edu. Or to: Evangelos Kiskinis, SF437, 7 Divinity Avenue, Cambridge, Massachusetts 02138, USA. Phone: (617) 496-8659; Fax: (617) 384-8234; E-mail: ekiskinis@mcb.harvard.edu.
1. Thomson JA, et al. Embryonic stem cell lines derived from human blastocysts. Science. 1998; 282(5391):1145-1147.

2. Takahashi $\mathrm{K}$, et al. Induction of pluripotent stem cells from adult human fibroblasts by defined factors. Cell. 2007;131(5):861-872.

3. Wakayama T, Perry AC, Zuccotti M, Johnson KR, Yanagimachi R. Full-term development of mice from enucleated oocytes injected with cumulus cell nuclei. Nature. 1998;394(6691):369-374.

4. Wilmut I, Schnieke AE, McWhir J, Kind AJ, Campbell KH. Viable offspring derived from fetal and adult mammalian cells. Nature. 1997; 385(6619):810-813.

5. Jaenisch R. Human cloning - the science and ethics of nuclear transplantation. $N$ Engl J Med. 2004;351(27):2787-2791.

6 . Byrne JA, et al. Producing primate embryonic stem cells by somatic cell nuclear transfer. Nature. 2007;450(7169):497-502.

7. Rideout WM III, Hochedlinger K, Kyba M, Daley GQ, Jaenisch R. Correction of a genetic defect by nuclear transplantation and combined cell and gene therapy. Cell. 2002;109(1):17-27.

8. Cowan CA, Atienza J, Melton DA, Eggan K. Nuclear reprogramming of somatic cells after fusion with human embryonic stem cells. Science. 2005; 309(5739):1369-1373.

9. Kanatsu-Shinohara M, et al. Generation of pluripotent stem cells from neonatal mouse testis. Cell. 2004;119(7):1001-1012.

10. Takahashi K, Yamanaka S. Induction of pluripotent stem cells from mouse embryonic and adult fibroblast cultures by defined factors. Cell. 2006;126(4):663-676

11. Tokuzawa Y, et al. Fbx15 is a novel target of Oct $3 / 4$ but is dispensable for embryonic stem cell selfrenewal and mouse development. Mol Cell Biol. 2003;23(8):2699-2708.

12. Maherali N, et al. Directly reprogrammed fibroblasts show global epigenetic remodeling and widespread tissue contribution. Cell Stem Cell. 2007; 1(1):55-70.

13. Meissner A, Wernig M, Jaenisch R. Direct reprogramming of genetically unmodified fibroblasts into pluripotent stem cells. Nat Biotechnol. 2007; 25(10):1177-1181

14. Okita K, Ichisaka T, Yamanaka S. Generation of germline-competent induced pluripotent stem cells. Nature. 2007;448(7151):313-317.

15 . Wernig $M$, et al. In vitro reprogramming of fibroblasts into a pluripotent ES-cell-like state. Nature. 2007;448(7151):318-324

16. Park IH, etal. Reprogramming of human somatic cells to pluripotency with defined factors. Nature. 2008; 451(7175):141-146.

17. Yu J, et al. Induced pluripotent stem cell lines derived from human somatic cells. Science. 2007; 318(5858):1917-1920.

18. Dimos JT, et al. Induced pluripotent stem cells generated from patients with ALS can be differentiated into motor neurons. Science. 2008;321(5893):1218-1221.

19. Park IH, et al. Disease-specific induced pluripotent stem cells. Cell. 2008;134(5):877-886.

20. Ebert AD, et al. Induced pluripotent stem cells from a spinal muscular atrophy patient. Nature. 2009; 457(7227):277-280.

21. Jaenisch R, Bird A. Epigenetic regulation of gene expression: how the genome integrates intrin- sic and environmental signals. Nat Genet. 2003 ; 33(suppl):245-254.

22. Hemberger M, Dean W, Reik W. Epigenetic dynamics of stem cells and cell lineage commitment: digging Waddington's canal. Nat Rev Mol Cell Biol. 2009;10(8):526-537.

23. Egli D, Birkhoff G, Eggan K. Mediators of reprogramming: transcription factors and transitions through mitosis. Nat Rev Mol Cell Biol. 2008;9(7):505-516.

24. Nichols J, et al. Formation of pluripotent stem cells in the mammalian embryo depends on the POU transcription factor Oct4. Cell. 1998;95(3):379-391.

25. Avilion AA, Nicolis SK, Pevny LH, Perez L, Vivian $\mathrm{N}$, Lovell-Badge R. Multipotent cell lineages in early mouse development depend on SOX2 function. Genes Dev. 2003;17(1):126-140.

26. Chambers I, et al. Nanog safeguards pluripotency and mediates germline development. Nature. 2007;450(7173):1230-1234.

27. Mitsui K, et al. The homeoprotein Nanog is required for maintenance of pluripotency in mouse epiblast and ES cells. Cell. 2003;113(5):631-642.

28 . Sridharan R, et al. Role of the murine reprogramming factors in the induction of pluripotency. Cell. 2009;136(2):364-377.

29. Stadtfeld M, Nagaya M, Utikal J, Weir G, Hochedlinger K. Induced pluripotent stem cells generated without viral integration. Science. 2008; 322(5903):945-949

30. Okita K, Nakagawa M, Hyenjong H, Ichisaka T, Yamanaka S. Generation of mouse induced pluripotent stem cells without viral vectors. Science. 2008;322(5903):949-953.

31. Zhou H, et al. Generation of induced pluripotent stem cells using recombinant proteins. Cell Stem Cell. 2009;4(5):381-384

32. Kim D, et al. Generation of human induced pluripotent stem cells by direct delivery of reprogramming proteins. Cell Stem Cell. 2009;4(6):472-476.

33. Yu J, et al. Human induced pluripotent stem cells free of vector and transgene sequences. Science. 2009;324(5928):797-801.

34. Hanna J, et al. Direct reprogramming of terminally differentiated mature B lymphocytes to pluripotency. Cell. 2008;133(2):250-264.

35. Mikkelsen TS, et al. Dissecting direct reprogram ming through integrative genomic analysis. Nature. 2008;454(7200):49-55.

36. Soldner F, et al. Parkinson's disease patient-derived induced pluripotent stem cells free of viral reprogramming factors. Cell. 2009;136(5):964-977.

37. Amabile G, Meissner A. Induced pluripotent stem cells: current progress and potential for regenerative medicine. Trends Mol Med. 2009;15(2):59-68.

38. Marion RM, et al. Telomeres acquire embryonic stem cell characteristics in induced pluripotent stem cells. Cell Stem Cell. 2009;4(2):141-154.

39. Chin $\mathrm{MH}$, et al. Induced pluripotent stem cells and embryonic stem cells are distinguished by gene expression signatures. Cell Stem Cell. 2009; 5(1):111-123.

40. Chen AE, et al. Optimal timing of inner cell mass isolation increases the efficiency of human embryonic stem cell derivation and allows generation of sibling cell lines. Cell Stem Cell. 2009;4(2):103-106.

41. Brambrink T, Hochedlinger K, Bell G, Jaenisch R. ES cells derived from cloned and fertilized blastocysts are transcriptionally and functionally indistinguish- able. Proc Natl Acad Sci U S A. 2006;103(4):933-938.

42. Boland $\mathrm{MJ}$, et al. Adult mice generated from induced pluripotent stem cells. Nature. 2009; 461(7260):91-94

43. Kang L, Wang J, Zhang Y, Kou Z, Gao S. iPS cells can support full-term development of tetraploid blastocyst-complemented embryos. Cell Stem Cell. 2009; 5(2):135-138

44. Zhao XY, et al. iPS cells produce viable mice through tetraploid complementation. Nature. 2009; 461(7260):86-90.

45. Maherali N, Hochedlinger K. Guidelines and techniques for the generation of induced pluripotent stem cells. Cell Stem Cell. 2008;3(6):595-605.

46. Aoi T, et al. Generation of pluripotent stem cells from adult mouse liver and stomach cells. Science. 2008; 321(5889):699-702.

47. Stadtfeld M, Brennand K, Hochedlinger K. Reprogramming of pancreatic beta cells into induced pluripotent stem cells. Curr Biol. 2008;18(12):890-894.

48. Eminli S, Utikal J, Arnold K, Jaenisch R, Hochedlinger K. Reprogramming of neural progenitor cells into induced pluripotent stem cells in the absence of exogenous Sox 2 expression. Stem Cells. 2008;26(10):2467-2474.

49. Aasen T, et al. Efficient and rapid generation of induced pluripotent stem cells from human keratinocytes. Nat Biotechnol. 2008;26(11):1276-1284.

50. Loh YH, et al. Generation of induced pluripotent stem cells from human blood. Blood. 2009; 113(22):5476-5479.

51 . Sun N, et al. Feeder-free derivation of induced pluripotent stem cells from adult human adipose stem cells. Proc Natl Acad Sci U S A. 2009;106(37):15720-15725

52. Utikal J, Maherali N, Kulalert W, Hochedlinger K. Sox 2 is dispensable for the reprogramming of melanocytes and melanoma cells into induced pluripotent stem cells. J Cell Sci. 2009;122(Pt 19):3502-3510.

53. Raya A, et al. Disease-corrected haematopoietic progenitors from Fanconi anaemia induced pluripotent stem cells. Nature. 2009; 460(7251):53-59.

54. Eminli S, et al. Differentiation stage determines potential of hematopoietic cells for reprogramming into induced pluripotent stem cells. Nat Genet. 2009;41(9):968-976.

55 . Miura K, et al. Variation in the safety of induced pluripotent stem cell lines. Nat Biotechnol. 2009; 27(8):743-745.

56. Huangfu D, et al. Induction of pluripotent stem cells by defined factors is greatly improved by small-molecule compounds. Nat Biotechnol. 2008;26(7):795-797.

57 . Huangfu D, et al. Induction of pluripotent stem cells from primary human fibroblasts with only Oct 4 and Sox2. Nat Biotechnol. 2008;26(11):1269-1275.

58. Shi Y, Do JT, Desponts C, Hahm HS, Scholer HR, Ding S. A combined chemical and genetic approach for the generation of induced pluripotent stem cells. Cell Stem Cell. 2008;2(6):525-528.

59. Lyssiotis CA, et al. Reprogramming of murine fibroblasts to induced pluripotent stem cells with chemical complementation of Klf4. Proc Natl Acad Sci U S A. 2009;106(22):8912-8917.

60. Shi Y, Desponts C, Do JT, Hahm HS, Scholer HR, Ding S. Induction of pluripotent stem cells from mouse embryonic fibroblasts by Oct 4 and Klf4 with small-molecule compounds. Cell Stem Cell. 2008; 3(5):568-574

61. Ichida JK, et al. A small-molecule inhibitor of Tgfbeta signaling replaces Sox 2 in reprogramming 
by inducing Nanog [published online ahead of print October 7, 2009]. Cell Stem Cell. doi:10.1016/ j.stem2009.09.012.

62. Carey BW, et al. Reprogramming of murine and human somatic cells using a single polycistronic vector. Proc Natl Acad Sci U S A. 2009;106(1):157-162.

63. Sommer CA, Stadtfeld M, Murphy GJ, Hochedlinger $\mathrm{K}$, Kotton DN, Mostoslavsky G. Induced pluripotent stem cell generation using a single lentiviral stem cell cassette. Stem Cells. 2009;27(3):543-549.

64. Hacein-Bey-Abina $S$, et al. Insertional oncogenesis in 4 patients after retrovirus-mediated gene therapy of SCID-X1. J Clin Invest. 2008;118(9):3132-3142.

65. Nakagawa M, et al. Generation of induced pluripotent stem cells without Myc from mouse and human fibroblasts. Nat Biotechnol. 2008;26(1):101-106.

66. Kaji K, Norrby K, Paca A, Mileikovsky M, Mohseni P, Woltjen K. Virus-free induction of pluripotency and subsequent excision of reprogramming factors. Nature. 2009;458(7239):771-775.

67 . Woltjen $\mathrm{K}$, et al. piggyBac transposition reprograms fibroblasts to induced pluripotent stem cells. Nature. 2009;458(7239):766-770.

68. Gonzalez F, et al. Generation of mouse-induced pluripotent stem cells by transient expression of a single nonviral polycistronic vector. Proc Natl Acad Sci U S A. 2009;106(22):8918-8922.

69. Daley GQ, Lensch MW, Jaenisch R, Meissner A, Plath $\mathrm{K}$, Yamanaka S. Broader implications of defining standards for the pluripotency of iPSCs. Cell Stem Cell. 2009;4(3):200-201; author reply 202.

70. Yamanaka S. A fresh look at iPS cells. Cell. 2009; 137(1):13-17.

71. Murry CE, Keller G. Differentiation of embryonic stem cells to clinically relevant populations: lessons from embryonic development. Cell. 2008; 132(4):661-680.

72. Lamba DA, Gust J, Reh TA. Transplantation of human embryonic stem cell-derived photoreceptors restores some visual function in Crx-deficient mice. Cell Stem Cell. 2009;4(1):73-79.

73. Yang D, Zhang ZJ, Oldenburg M, Ayala M, Zhang SC. Human embryonic stem cell-derived dopaminergic neurons reverse functional deficit in parkinsonian rats. Stem Cells. 2008;26(1):55-63.

74. Kim JH, et al. Dopamine neurons derived from embryonic stem cells function in an animal model of Parkinson's disease. Nature. 2002;418(6893):50-56.

75. Keirstead HS, et al. Human embryonic stem cellderived oligodendrocyte progenitor cell transplants remyelinate and restore locomotion after spinal cord injury. J Neurosci. 2005;25(19):4694-4705.

76. Sharp J, Keirstead HS. Stem cell-based cell replacement strategies for the central nervous system. Neurosci Lett. 2009;456(3):107-111.

77. Hanna J, et al. Treatment of sickle cell anemia mouse model with iPS cells generated from autologous skin. Science. 2007;318(5858):1920-1923.

78. Zwaka TP, Thomson JA. Homologous recombination in human embryonic stem cells. Nat Biotechnol. 2003;21(3):319-321.

79. Zou J, et al. Gene targeting of a disease-related gene in human induced pluripotent stem and embryonic stem cells. Cell Stem Cell. 2009;5(1):97-110.

80 . Wernig $M$, et al. Neurons derived from reprogrammed fibroblasts functionally integrate into the fetal brain and improve symptoms of rats with Parkinson's disease. Proc Natl Acad Sci U S A. 2008; 105(15):5856-5861.

81. Xu D, et al. Phenotypic correction of murine hemophilia A using an iPS cell-based therapy. Proc Natl Acad Sci U S A. 2009;106(3):808-813.

82. Dunckley T, et al. Whole-genome analysis of sporadic amyotrophic lateral sclerosis. $N$ Engl J Med. 2007;357(8):775-788.

83. Johnson FO, Atchison WD. The role of environmental mercury, lead and pesticide exposure in development of amyotrophic lateral sclerosis. Neurotoxicology. 2009;30(5):761-765.

84. Migliore L, Coppede F. Genetics, environmental factors and the emerging role of epigenetics in neurodegenerative diseases. Mutat Res. 2009;667(1-2):82-97.

85. Piekarz RL, Bates SE. Epigenetic modifiers: basic understanding and clinical development. Clin Cancer Res. 2009;15(12):3918-3926.

86. Lee G, et al. Modelling pathogenesis and treatment of familial dysautonomia using patient-specific iPSCs. Nature. 2009;461(7262):402-406

87. Rubin LL. Stem cells and drug discovery: the beginning of a new era? Cell. 2008;132(4):549-552.

88. Friedrich Ben-Nun I, Benvenisty N. Human embryonic stem cells as a cellular model for human disor- ders. Mol Cell Endocrinol. 2006;252(1-2):154-159.

89. Di Giorgio FP, Carrasco MA, Siao MC, Maniatis T, Eggan K. Non-cell autonomous effect of glia on motor neurons in an embryonic stem cell-based ALS model. Nat Neurosci. 2007;10(5):608-614.

90. Nagai M, et al. Astrocytes expressing ALS-linked mutated SOD1 release factors selectively toxic to motor neurons. Nat Neurosci. 2007;10(5):615-622.

91. Yamashita H, et al. Embryonic stem cell-derived neuron models of Parkinson's disease exhibit delayed neuronal death. J Neurochem. 2006;98(1):45-56.

92. Eiges R, et al. Developmental study of fragile X syndrome using human embryonic stem cells derived from preimplantation genetically diagnosed embryos. Cell Stem Cell. 2007;1(5):568-577.

93. Happe F, Ronald A, Plomin R. Time to give up on a single explanation for autism. Nat Neurosci. 2006;9(10):1218-1220.

94. Maehr R, et al. Generation of pluripotent stem cells from patients with type 1 diabetes. Proc Natl Acad Sci U S A. 2009;106(37):15768-15773.

95. Di Giorgio FP, Boulting GL, Bobrowicz S, Eggan KC. Human embryonic stem cell-derived motor neurons are sensitive to the toxic effect of glial cells carrying an ALS-causing mutation. Cell Stem Cell. 2008;3(6):637-648.

96. Marchetto MC, Muotri AR, Mu Y, Smith AM, Cezar GG, Gage FH. Non-cell-autonomous effect of human SOD1 G37R astrocytes on motor neurons derived from human embryonic stem cells. Cell Stem Cell. 2008;3(6):649-657.

97. Davila JC, Cezar GG, Thiede M, Strom S, Miki T, Trosko J. Use and application of stem cells in toxicology. Toxicol Sci. 2004;79(2):214-223.

98. Wernig M, Meissner A, Cassady JP, Jaenisch R. $\mathrm{c}-\mathrm{Myc}$ is dispensable for direct reprogramming of mouse fibroblasts. Cell Stem Cell. 2008;2(1):10-12.

99. Kim JB, et al. Pluripotent stem cells induced from adult neural stem cells by reprogramming with two factors. Nature. 2008;454(7204):646-650.

100. Kim JB, et al. Oct4-induced pluripotency in adult neural stem cells. Cell. 2009;136(3):411-419.

101. Maherali N, Ahfeldt T, Rigamonti A, Utikal J, Cowan $\mathrm{C}$, Hochedlinger K. A high-efficiency system for the generation and study of human induced pluripotent stem cells. Cell Stem Cell. 2008;3(3):340-345. 